\title{
LEBESGUE'S THEOREM OF DIFFERENTIATION IN FRÉCHET LATTICES
}

\author{
KARL-GOSWIN GROSSE-ERDMANN \\ (Communicated by Palle E. T. Jorgensen)
}

\begin{abstract}
Lebesgue's differentiation theorem (LDT) states that every monotonic real function is differentiable a.e. We investigate the validity of this theorem for functions with values in topological vector lattices. It is shown that a Fréchet lattice satisfies (LDT) iff it is isomorphic to a generalized echelon space, a Banach lattice satisfies (LDT) iff it is isomorphic to some $l^{1}(\Gamma)$.
\end{abstract}

\section{INTRODUCTION}

By a well-known theorem of Lebesgue every monotonic real function is differentiable almost everywhere. The same then holds for every function of bounded variation. First attempts were made in the 1930's to generalize the latter result to functions with values in a Banach space. It turned out that not every Banach space allows such a generalization. This led to the concept of the Gel'fand property and later to that of the Radon-Nikodým property.

Consider, more generally, a locally convex space $X$. A function $F:[0,1] \rightarrow$ $X$ is of bounded variation if for each continuous seminorm $p$ on $X$ the $p$ variation $V_{p} F$ of $F$ is finite, where $V_{p} F=\sup \left\{\sum_{n=0}^{N-1} p\left(F\left(t_{n+1}\right)-F\left(t_{n}\right)\right)\right.$ : $\left.0=t_{0}<\cdots<t_{N}=1, N \in \mathbb{N}\right\}$. The space $X$ is then said to have the Gel' fand property if every function $F:[0,1] \rightarrow X$ of bounded variation is differentiable almost everywhere. It is well known that for Banach spaces the Gel'fand property is equivalent to the Radon-Nikodým property. For details see Diestel and Uhl [1].

In this note we consider (real) topological vector spaces that have an additional compatible order structure. For these spaces one may investigate the validity of Lebesgue's original observation: Is every monotonic function from $[0,1]$ into such a space differentiable almost everywhere? We show for a large class of locally convex vector lattices that Lebesgue's theorem holds iff the space is a generalized AL-space (see $\S 2$ ) with Gel'fand property. Moreover the space

Received by the editors March 6, 1989.

1980 Mathematics Subject Classification (1985 Revision). Primary 26E20, 46A40; Secondary 46A45, 46B30.

Key words and phrases. Differentiation, monotonic functions, Gel' fand property, locally convex vector lattices, generalized AL-spaces, Köthe spaces, Köthe sequence spaces. 
has to be discrete. In particular, Lebesgue's theorem holds in a Fréchet lattice iff it is isomorphic to an echelon space (gestufter Raum) with possibly uncountably many coordinates, it holds in a Banach lattice iff it is isomorphic to some $l^{1}(\Gamma)$.

\section{Generalized AL-SPaces}

A topological vector lattice is a vector lattice (Riesz space) with a vector space topology in which its positive cone is closed. A seminorm $p$ on a vector lattice is called a Riesz seminorm if $p(x) \leq p(y)$ for $|x| \leq|y|$. A locally convex vector lattice is a topological vector lattice whose topology is induced by a separating family of Riesz seminorms (i.e., we assume the space to be locally solid). A complete locally convex vector lattice is a Fréchet lattice if this family can be chosen countable, a Banach lattice if a single Riesz norm suffices. In this paper we understand isomorphisms as isomorphisms between topological vector lattices if nothing else is said.

The concept of AL-spaces for Banach lattices has a natural generalization to locally convex vector lattices (cf. Wong and $\mathrm{Ng}$ [14]):

Definition. A locally convex vector lattice $E$ is a generalized AL-space if its topology can be defined by a family of Riesz seminorms $p$ that are additive on the positive cone, i.e., with $p(x+y)=p(x)+p(y)$ for $x, y \in E^{+}$.

Note that a Banach lattice is a generalized AL-space iff it is isomorphic to an AL-space.

For a vector lattice $E$ and a separating solid subspace $F$ of the order dual $E^{\sim}$ the topology $|\sigma|(E, F)$ on $E$ is defined by the Riesz seminorms $p_{f}(x)=$ $|f|(|x|)$ for $x \in E(f \in F)$. Conversely, given a topological vector lattice $E$ and a continuous Riesz seminorm $p$ on $E$ that is additive on $E^{+}$, then $f_{p}(x)=p\left(x^{+}\right)-p\left(x^{-}\right)$for $x \in E$ defines a continuous positive linear functional on $E$, i.e., a positive element of the topological dual $E^{\prime}$. Consequently we have (cf. Wong [13, 4.2]):

Theorem 1. Let $E$ be a locally convex vector lattice. Then the following are equivalent:

(1) $E$ is a generalized AL-space;

(2) the topology of $E$ coincides with $|\sigma|\left(E, E^{\prime}\right)$;

(3) for each continuous Riesz seminorm $p$ on $E$ there is a continuous positive linear functional $f$ on $E$ with $p(x) \leq f(x)$ for $x \in E^{+}$.

As in the case of Banach lattices, complete generalized AL-spaces have very strong properties. As defined in [5], the topology of a topological vector lattice $E$ is called Levi if each set in $E$ that is directed upwards and bounded (in this topology) is order bounded; it is called Lebesgue if for each set $A$ in $E$ that is directed upwards and has a supremum (the filter of sections of) $A$ converges to $\sup A$. For a vector lattice $E$ the dual $E^{\times}$is defined as the 
set of all functionals on $E$ that are the difference of two order-continuous positive linear functionals. A vector lattice $E$ is called perfect if the canonical map ${ }^{\sim}: E \rightarrow\left(E^{\times}\right)^{\times}, \tilde{x}(f)=f(x)$ for $x \in E, f \in E^{\times}$is a vector lattice isomorphism. We have:

Theorem 2. Let $E$ be a complete locally convex vector lattice that is a generalized AL-space. Then $E$ has the following properties:

(1) The topology of $E$ is Levi and Lebesgue.

(2) $E$ is order complete.

(3) $E$ is perfect.

Proof. (1) and (2) follow as in the case of Banach lattices, see [9, II.8.2/3]. (3) then follows with [5, 33E].

We want to show that every complete generalized AL-space has a representation as a certain function space. Let $X$ be a locally compact Hausdorff topological space and $\mu$ a Radon measure on a $\sigma$-algebra $\Sigma$ in $X$ in the sense of $[5,73 \mathrm{~A}]$. Then $\Omega(X, \Sigma, \mu)=\Omega(\mu)$ is defined as the space of (equivalence classes of) locally integrable real functions on $X$, i.e., of measurable functions $f: X \rightarrow \mathbb{R}$ that are integrable on every compact subset of $X . \Omega(\mu)$ is a vector lattice in its canonical order. Define $\Phi(\mu)$ as the subspace of $\Omega(\mu)$ of (equivalence classes of) essentially bounded functions with compact support. The $\alpha$-dual of a subspace $L$ of $\Omega(\mu)$ is defined as

$$
L^{\alpha}=\left\{g \in \Omega(\mu): \int|f \cdot g| d \mu<\infty \text { for each } f \in L\right\} .
$$

$L^{\alpha}$ is called a Köthe space. Since $L^{\alpha \alpha \alpha}=L^{\alpha}$, a subspace $M$ of $\Omega(\mu)$ is a Köthe space iff $M^{\alpha \alpha}=M$. Fremlin ([4, Theorem 7], see also [5, 65A]) showed that for a solid subspace $L$ of $\Omega(\mu)$ that contains $\Phi(\mu)$ one can identify $L^{\times}$ with $L^{\alpha}$ via $\Lambda_{g}(f)=\int(f \cdot g) d \mu$ for $f \in L$. Hence a subspace $L$ of $\Omega(\mu)$ is a Köthe space iff it is a perfect solid subspace that contains $\Phi(\mu)$. With the help of a representation theorem due to Fremlin [4] we obtain:

Theorem 3. Let $E$ be a locally convex vector lattice. Then $E$ is a complete generalized $\mathrm{AL}$ - space iff it is isomorphic to a Köthe space $L^{\alpha}$ under the topology $|\sigma|\left(L^{\alpha}, L\right)$, where $L$ is a solid subspace of some $\Omega(\mu)$ containing $\Phi(\mu)$ with $\mu$ a Radon measure on a locally compact Hausdorff topological space.

Proof. For a solid subspace $L$ of $\Omega(\mu)$ with $\Phi(\mu) \subset L$ one can identify $L^{\alpha}$ with $L^{\times}$. Hence the space $\left(L^{\alpha},|\sigma|\left(L^{\alpha}, L\right)\right)$ is clearly a generalized AL-space and complete by [5, 81D].

Conversely, let $E$ be a complete generalized AL-space. Since the topology of $E$ is Lebesgue, we have $E^{\prime} \subset E^{\times} \subset E^{\sim}$. Thus $E^{\prime}$, being a solid subspace of the order complete space $E^{\sim}$, is order complete. Also $E \simeq E^{\times \times} \subset E^{\prime \times}$, hence $E^{\prime \times}$ separates the points of $E^{\prime}$. By [4, Theorem 6] there now is a Radon measure $\mu$ on a locally compact Hausdorff topological space such that $E^{\prime}$ is a vector 
lattice isomorphic to a solid subspace $L$ of $\Omega(\mu)$ with $\Phi(\mu) \subset L$, and $E^{\prime \times}$ can be identified with $L^{\alpha}$. By [5,32C], $E$ can be identified with an order-dense solid subspace $M$ of $L^{\alpha}$. Since the topology of $E$ equals $|\sigma|\left(E, E^{\prime}\right)$ and is complete, it follows that $E$ is isomorphic to $M$ considered as a subspace of $L^{\alpha}$ under the topology $|\sigma|\left(L^{\alpha}, L\right)$ and that $M$ is closed in $L^{\alpha}$. Hence $M=L^{\alpha}$ because $M$ is order-dense and $|\sigma|\left(L^{\alpha}, L\right)$ is Lebesgue. Thus $E$ is isomorphic to $L^{\alpha}$ under $|\sigma|\left(L^{\alpha}, L\right)$.

Consider an order complete vector lattice $E$. An element $x \neq 0$ of $E$ is called an atom if $|x|=u+v, u, v \geq 0$ and $u \wedge v=0$ implies $u=0$ or $v=0$. The space $E$ is called discrete if $E$ coincides with the band generated by its atoms. Consider in particular a Köthe space $L$ in some $\Omega(X, \Sigma, \mu)$. $L$ contains the characteristic function $\chi_{K}$ of every compact subset $K$ of $X$. Thus $L$ is seen to be discrete iff $\mu$ is a purely atomic measure, i.e., iff every measurable set of positive measure contains a $\mu$-atom. It follows that $L$ is discrete iff it is vector lattice isomorphic to a Köthe space in some $\omega(\Gamma):=$ $\mathbb{R}^{\Gamma}=\Omega(\Gamma, \tau, \nu)$, where $\Gamma$ is a nonempty set carrying the discrete topology $\tau$ and $\nu$ is the counting measure. We have $\varphi(\Gamma):=\Phi(\nu)=\left\{\left(x_{1}\right) \in \omega(\Gamma): x_{1} \neq 0\right.$ only for finitely many $l\}$. A Köthe space in some $\omega(\Gamma)$ is called a Köthe sequence space (even if $\Gamma$ is uncountable). Thus we have:

Theorem 4. Let $E$ be a locally convex vector lattice. Then $E$ is a complete discrete generalized $\mathrm{AL}-$ space iff it is isomorphic to a Köthe sequence space $\lambda^{\alpha}$ under the topology $|\sigma|\left(\lambda^{\alpha}, \lambda\right)$, where $\lambda$ is a solid subspace of some $\omega(\Gamma)$ containing $\varphi(\Gamma)$, with some nonempty set $\Gamma$.

For Banach lattices Schlotterbeck ([10], see also [9, IV.2.7]) characterized ALspaces as those in which every convergent series of positive elements converges absolutely. This can be generalized to spaces in which every positive linear functional is continuous. Examples of such spaces can be found in [8, V.5.5]; they include all complete bornological vector lattices, hence all Fréchet lattices.

Theorem 5. Let $E$ be a locally convex vector lattice in which every positive linear functional is continuous. Then the following are equivalent:

(1) $E$ is a generalized AL-space;

(2) every convergent series $\sum_{n=1}^{\infty} x_{n}$ of positive elements of $E$ converges absolutely, that is, $\sum_{n=1}^{\infty} p\left(x_{n}\right)<\infty$ for each continuous seminorm $p$ on $E$.

Proof. (1) $\Rightarrow(2)$. Suppose that $\sum_{n=1}^{\infty} x_{n}$ converges, with $x_{n} \in E^{+}$. Each continuous seminorm $p$ on $E$ is dominated by a continuous Riesz seminorm $q$ that is additive on $E^{+}$. Thus $\sum_{n=1}^{N} p\left(x_{n}\right) \leq q\left(\sum_{n=1}^{N} x_{n}\right)$ is bounded as $N \rightarrow \infty$.

(2) $\Rightarrow(1)$. Let $p$ be a Riesz seminorm on $E$ and put

$$
\tilde{p}(x)=\sup \left\{\sum_{n=1}^{N} p\left(x_{n}\right): x=\sum_{n=1}^{N} x_{n}, x_{n} \in E^{+}, N \in \mathbb{N}\right\}
$$


for $x \in E^{+}$. Then $\tilde{p}(x)$ is finite; else there are $x_{\nu}^{(n)} \in E^{+}$with $2^{-n} x=$ $\sum_{\nu=1}^{N_{n}} x_{\nu}^{(n)}$ and $\sum_{\nu=1}^{N_{n}} p\left(x_{\nu}^{(n)}\right) \geq 1$, and $\left(\xi_{n}\right)=\left(x_{1}^{(1)}, \ldots, x_{N_{1}}^{(1)}, x_{1}^{(2)}, \ldots\right)$ has a convergent series with $\sum_{n=1}^{\infty} p\left(\xi_{n}\right)=\infty$. Furthermore we clearly have $\tilde{p}(x) \geq$ $0, \tilde{p}(x+y) \geq \tilde{p}(x)+\tilde{p}(y)$, and $\tilde{p}(\alpha x)=\alpha \tilde{p}(x)$ for $x, y \in E^{+}$and $\alpha \geq 0$. By the decomposition property $[9$, II.1.6] we also have $\tilde{p}(x+y) \leq \tilde{p}(x)+\tilde{p}(y)$ for $x, y \in E^{+}$. Setting $f(x)=\tilde{p}\left(x^{+}\right)-\tilde{p}\left(x^{-}\right)$for $x \in E, \tilde{p}$ can be extended to a positive linear hence continuous functional $f$ on $E$. Moreover we have $f(x)=\tilde{p}(x) \geq p(x)$ for $x \in E^{+}$. By Theorem $1, E$ is a generalized ALspace.

Under different assumptions this result can be found in Walsh [12, Corollary 3.2.8] and Wong [13, 3.3].

\section{LEBESGUE'S DIFFERENTIATION THEOREM}

With the above characterization of generalized AL-spaces we obtain:

Theorem 6. Let $E$ be a complete locally convex vector lattice in which every positive linear functional is continuous. Then the following are equivalent:

(1:LDT) Every monotonic function $F:[0,1] \rightarrow E$ is differentiable a.e.;

(2) if $F:[0,1] \rightarrow E$ is a function for which the set

$$
\left\{\sum_{n=0}^{N-1}\left|F\left(t_{n+1}\right)-F\left(t_{n}\right)\right|: 0=t_{0}<\cdots<t_{N}=1, N \in \mathbb{N}\right\}
$$

is order bounded, then $F$ is differentiable a.e.;

(3) if $F:[0,1] \rightarrow E$ is a function for which the set

$$
\left\{\sum_{n=0}^{N-1}\left|F\left(t_{n+1}\right)-F\left(t_{n}\right)\right|: 0=t_{0}<\cdots<t_{N}=1, N \in \mathbb{N}\right\}
$$

is bounded (in the topology), then $F$ is differentiable a.e.;

(4) E is a generalized AL-space with Gel'fand property.

Proof. (1) $\Rightarrow(4)$. Assume that $E$ is not a generalized AL-space. Then there is by Theorem 5 a sequence $\left(x_{n}\right)$ in $E^{+}$such that $\sum_{n=1}^{\infty} x_{n}$ converges but $\sum_{n=1}^{\infty} p\left(x_{n}\right)=\infty$ for some continuous seminorm $p$. We can assume $p\left(x_{n}\right)>0$ for $n \in \mathbb{N}$. Let $\left(\alpha_{n}\right)$ be a positive sequence with $\alpha_{n} \rightarrow 0$ and $\sum_{n=1}^{\infty} \alpha_{n} p\left(x_{n}\right)=$ $\infty$. Consider for $n \in \mathbb{N}$ the set

$$
A_{n}=\left\{t=\tau-[\tau] \in[0,1): \sum_{\nu=1}^{n-1} \alpha_{\nu} p\left(x_{\nu}\right) \leq \tau<\sum_{\nu=1}^{n} \alpha_{\nu} p\left(x_{\nu}\right)\right\}
$$

and define $F:[0,1] \rightarrow E$ by

$$
F(t)=\sum_{n=1}^{\infty} \frac{\lambda\left([0, t) \cap A_{n}\right)}{\lambda\left(A_{n}\right)} \cdot x_{n}
$$


where $\lambda$ is the Lebesgue measure. Since the coefficients of the $x_{n}$ lie in $[0,1]$, $F(t)$ exists for every $t \in[0,1] . F$ is clearly monotonic. Now fix a $t \in[0,1)$. Since $\sum_{n=1}^{\infty} \alpha_{n} p\left(x_{n}\right)=\infty$, there is a sequence $\left(n_{k}\right)$ with $t \in A_{n_{k}}$ for $k \in \mathbb{N}$. Moreover, there are $\delta_{k}>0$ with $\left[t, t+\delta_{k}\right) \subset A_{n_{k}}$ for $k \in \mathbb{N}$. Hence we have for $0<d<\delta_{k}$ :

$$
\begin{aligned}
p\left(\frac{F(t+d)-F(t)}{d}\right) & \geq p\left(\frac{\lambda\left([t, t+d) \cap A_{n_{k}}\right)}{d \cdot \lambda\left(A_{n_{k}}\right)} \cdot x_{n_{k}}\right) \\
& =p\left(\frac{x_{n_{k}}}{\lambda\left(A_{n_{k}}\right)}\right) \geq \frac{1}{\alpha_{n_{k}}}
\end{aligned}
$$

with $1 / \alpha_{n_{k}} \rightarrow \infty$ as $k \rightarrow \infty$. Thus, $F$ is nowhere differentiable on $[0,1)$, which is a contradiction. So $E$ is a generalized AL-space. Now let $F:[0,1] \rightarrow$ $E$ be a function of bounded variation. Then the set $\left\{\sum_{n=0}^{N-1}\left|F\left(t_{n+1}\right)-F\left(t_{n}\right)\right|\right.$ : $\left.0=t_{0}<\cdots<t_{N}=1, N \in \mathbb{N}\right\}$ is directed upwards and bounded in the vector space topology of $E$, hence order bounded by Theorem 2.(1). We also have that $E$ is order complete. It now follows from a theorem of Faires and Morrison [3, Theorem 1] that $F$ is the difference of two monotonic functions. Hence $F$ is differentiable almost everywhere and $E$ has the Gel'fand property.

(4) $\Rightarrow(3)$. In a generalized AL-space every function $F:[0,1] \rightarrow E$ as in (3) is of bounded variation and hence differentiable almost everywhere by the Gel'fand property.

(3) $\Rightarrow(2) \Rightarrow(1)$ is trivial.

We show that discreteness of the vector lattice is another necessary condition for (LDT). For a Köthe space $L$ the topology $|\sigma|\left(L, L^{\alpha}\right)$ is called its normal topology.

Theorem 7. Let $E$ be a complete locally convex vector lattice in which every positive linear functional is continuous. If $E$ satisfies (LDT), then it is a discrete generalized AL-space and hence is isomorphic to a Köthe sequence space under its normal topology.

Proof. 1. If $E$ satisfies (LDT), it is a generalized AL-space by Theorem 6 . Assume that $E$ is not discrete. Then there is a point $a>0$ in $E$ that is orthogonal to the band generated by the atoms of $E$. Let $f$ be a continuous positive linear functional on $E$ with $f(a)=1$. By Theorem 2, $E$ is order complete and its topology is Lebesgue. Hence we can assume that $f(x)>0$ for $0<x \leq a$. Since $[0, a]$ does not contain any atoms, a standard argument now shows the existence of points $a_{1,1}$ and $a_{1,2}$ in $[0, a]$ with $f\left(a_{1,1}\right)=$ $f\left(a_{1,2}\right)=\frac{1}{2}, a_{1,1}+a_{1,2}=a$, and $a_{1,1} \wedge a_{1,2}=0$. By induction we obtain elements $a_{n, \nu}$ in $[0, a], n \in \mathbb{N}$ and $\nu=1, \ldots, 2^{n}$, with $f\left(a_{n, \nu}\right)=2^{-n}$, $a_{n, \nu} \wedge a_{n, \mu}=0$ for $\nu \neq \mu$ and $a_{n+1,2 \kappa-1}+a_{n+1,2 \kappa}=a_{n, \kappa}$ for $n \in \mathbb{N}$ and $\kappa=1, \ldots, 2^{n}$. 
2. We use (1) to imitate the nondifferentiability of $F:[0,1] \rightarrow L^{1}([0,1])$, $F(t)=\chi_{[0, t)}$. Define $F:[0,1] \rightarrow E$ by $F(t)=\sup \left\{\sum_{\nu=1}^{m} a_{n, \nu}: m, n \in \mathbb{N}\right.$, $\left.m / 2^{n}<t\right\} . \quad F$ is well defined and $F(t)$ equals the limit of the respective directed set. $F$ is clearly monotonic. Since $|x| \wedge|y|=0$ implies $|x+y|=$ $|x|+|y|$ for $x, y \in E$, we have

$$
\begin{aligned}
f\left(\left|\frac{F(t+h)-F(t)}{h}-\frac{F\left(t+h^{\prime}\right)-F(t)}{h^{\prime}}\right|\right) & =\int_{0}^{1}\left|\frac{\chi_{[t, t+h)}(x)}{h}-\frac{\chi_{\left[t, t+h^{\prime}\right)}(x)}{h^{\prime}}\right| d x \\
& =2\left(1-\frac{h}{h^{\prime}}\right)
\end{aligned}
$$

for $t \in[0,1)$ and sufficiently small $h, h^{\prime}$ with $0<h<h^{\prime}$. Hence $F$ is nowhere differentiable on $[0,1)$, which contradicts (LDT).

3. Thus $E$ is a discrete complete generalized AL-space. By Theorem 4, $E$ is isomorphic to a Köthe sequence space $\lambda$ under the topology $|\sigma|(\lambda, \mu)$, where $\mu$ is a subspace of $\lambda^{\alpha}$ with $\mu=\lambda^{\prime}$. By assumption $\lambda^{\alpha} \subset \lambda^{\alpha}$ is contained in $\lambda^{\prime}$, hence $\mu=\lambda^{\alpha}$. Thus $\lambda$ carries its normal topology.

Remark. The condition given in the theorem is not sufficient in general (it is, however, for Fréchet lattices; see infra). To see this consider the space $\omega(I)=$ $\mathbb{R}^{I}$ with $I=[0,1]$. Its product topology coincides with $|\sigma|(\omega(I), \varphi(I))$. Note that $\omega(I)^{\alpha}=\varphi(I)$ and $\varphi(I)^{\alpha}=\omega(I)$. However the monotonic function $F:[0,1] \rightarrow \omega(I), F(t)=\chi_{[0, t)}$ is differentiable for no $t \in[0,1)$.

\section{Fréchet lattices With (LDT)}

Theorem 7 leads to a representation of Fréchet lattices with (LDT) as echelon spaces. Let $P=\left(\left(y_{l}^{(n)}\right)_{l \in \Gamma}\right)_{n \in \mathbb{N}}$ be a sequence of positive elements in a sequence space $\omega(\Gamma)$ with the property that to each $l \in \Gamma$ there is an $n \in \mathbb{N}$ with $y_{l}^{(n)}>0$. The (generalized) echelon space $\lambda(P)$ is then defined as

$$
\lambda(P)=\left\{\left(x_{l}\right) \in \omega(\Gamma): \sum_{l}\left|x_{l}\right| y_{l}^{(n)}<\infty \text { for each } n \in \mathbb{N}\right\},
$$

endowed with the topology given by the seminorms $p_{n}\left(\left(x_{l}\right)\right)=\sum_{l}\left|x_{l}\right| y_{l}^{(n)}, n \in$ $\mathbb{N}$ (cf. [7, §30.8]). It is not difficult to see that the echelon spaces are exactly the Köthe sequence spaces that are Fréchet under their normal topology and that a normable echelon space is isomorphic to $l^{1}(\Gamma)$.

Theorem 8. A Fréchet lattice satisfies (LDT) iff it is isomorphic to an echelon space. A Banach lattice satisfies (LDT) iff it is isomorphic to $l^{1}(\Gamma)$ for some nonempty set $\Gamma$.

Proof. The necessity is clear by our last remark. Conversely, let $\lambda(P)$ be an echelon space with $P=\left(\left(y_{l}^{(n)}\right)\right)$ and $E_{n}$ the completion of the quotient $\lambda(P) / N_{n}$ under the topology induced by $p_{n}$, where $N_{n}=\left\{\left(x_{l}\right): \sum_{l}\left|x_{l}\right| y_{l}^{(n)}=0\right\}$ and 
$p_{n}\left(\left(x_{l}\right)\right)=\sum_{l}\left|x_{l}\right| y_{l}^{(n)}, n \in \mathbb{N}$. Then $E_{n}$ is isomorphic to $l^{1}\left(\Gamma_{n}\right)$ with $\Gamma_{n}=\{l$ : $\left.y_{l}^{(n)} \neq 0\right\}$ and $\lambda(P)$ is isomorphic to a closed linear sublattice of $\prod_{n} l^{1}\left(\Gamma_{n}\right)$. Since each $l^{1}\left(\Gamma_{n}\right)$ is an AL-space that has the Radon-Nikodým property, hence the Gel'fand property, it satisfies (LDT) by Theorem 6 . The same then holds for $E$.

For closely related characterizations of $l^{1}(\Gamma)$ in terms of convergence of certain (sub)martingales see Szulga [11] and Egghe [2].

Kōmura and Koshi [6] showed that every nuclear locally convex vector lattice is isomorphic to a Köthe sequence space $(\lambda,|\sigma|(\lambda, \mu))$, where $\mu$ is a subspace of $\lambda^{\alpha}$ with $\mu=\lambda^{\prime}$. Thus we have (see the proof of Theorem 7):

Corollary. Every nuclear Fréchet lattice satisfies (LDT).

This need not hold if the space is not Fréchet, as $\omega([0,1])$ shows.

Additional remark. Wong and $\mathrm{Ng}[14, \mathrm{p}$. 55] ask whether a locally convex vector lattice is nuclear if it is a generalized AL-space in which every convergent series is the difference of two convergent series of positive terms. The space $(\varphi(\Gamma),|\sigma|(\varphi(\Gamma), \omega(\Gamma)))$ with any uncountable set $\Gamma$ provides a counterexample. This space is not nuclear (use [6, Lemma 3]), but every series lies in some nuclear subspace $(\varphi(I),|\sigma|(\varphi(I), \omega(I)))$ with $I \subset \Gamma$ countable.

\section{REFERENCES}

1. J. Diestel and J. J. Uhl, Vector measures, Math. Surveys, no. 15, Amer. Math. Soc., Providence, RI, 1977.

2. L. Egghe, On sub-and superpramarts with values in a Banach lattice, Measure Theory Oberwolfach 1981 (Oberwolfach 1981), Lecture Notes in Math., vol. 945, Springer-Verlag, Berlin, Heidelberg, and New York, 1982, pp. 352-365.

3. B. Faires and T. J. Morrison, The Jordan decomposition of vector-valued measures, Proc. Amer. Math. Soc. 60 (1976), 139-143.

4. D. H. Fremlin, Abstract Köthe spaces. II, Proc. Cambridge Philos. Soc. 63 (1967), 951-956.

5. $\ldots$, Topological Riesz spaces and measure theory, Cambridge Univ. Press, Cambridge, 1974.

6. Y. Kōmura and S. Koshi, Nuclear vector lattices, Math. Ann. 163 (1966), 105-110.

7. G. Köthe, Topological vector spaces. I, Springer-Verlag, Berlin, Heidelberg, and New York, 1969.

8. H. H. Schaefer, Topological vector spaces, Springer-Verlag, New York, Heidelberg, and Berlin, 1971.

9. __ Banach lattices and positive operators, Springer-Verlag, New York, Heidelberg, and Berlin, 1974.

10. U. Schlotterbeck, Über Klassen majorisierbarer Operatoren auf Banachverbänden, Dissertation, Tübingen, 1969.

11. J. Szulga, On the submartingale characterization of Banach lattices isomorphic to $l_{1}$, Bull. Acad. Polon. Sci. Sér. Sci. Math. Astronom. Phys. 26 (1978), 65-68.

12. B. Walsh, Ordered vector sequence spaces and related classes of linear operators, Math. Ann. 206 (1973), 89-138. 
13. Y. C. Wong, Characterizations of the topology of uniform convergence on order-intervals, Hokkaido Math. J. 5 (1976), 164-200.

14. Y. C. Wong and K. F. Ng, Nuclear spaces and generalized AL-spaces, Southeast Asian Bull. Math. 5 (1981), 45-58.

Fachbereich IV - Mathematik, Universität Trier, Postfach 3825, 5500 Trier, West GERMANY

Current address: Fachbereich Mathematik, FernUniversität Hagen, Postfach 940, D-5800 Hagen, West Germany 